\title{
LARGE TIME BEHAVIOR FOR THE POROUS MEDIUM EQUATION WITH CONVECTION
}

\author{
DANIELE ANDREUCCI AND ANATOLI F. TEDEEV
}

\begin{abstract}
We consider the Cauchy problem for the porous medium equation with nonlinear convection, when the nonlinearities are the same in the convection and in the diffusion terms. We get a new sharp bound of the solution for large times.

KEYWORDS: convection-diffusion equation, large time behavior, sup estimate, entropy inequality

This is a post-peer-review, pre-copyedit version of an article published in Meccanica 52 (2017), no. 13, 3255-3260. The final authenticated version is available online at: https:doi-org.ezproxy.uniroma1.it/10.1007/s11012017-0624-2.
\end{abstract}

\section{INTRODUCTION}

We consider the Cauchy problem for the porous medium equation with convection in the form

$$
\begin{aligned}
\frac{\partial u}{\partial t} & =\Delta u^{m}-\left(u^{m}\right)_{x_{N}}, & & (x, t) \in \boldsymbol{R}^{N} \times(0,+\infty), \\
u(x, 0) & =u_{0}(x), & & x \in \boldsymbol{R}^{N} .
\end{aligned}
$$

Here we assume $m>1, N \geq 2, u_{0} \in L^{1}\left(\boldsymbol{R}^{N}\right), u_{0} \geq 0$.

Theorem 1.1. There exists a non-negative weak solution u of (1)-(2) such that for all $t>0$

$$
\|u(t)\|_{L^{\infty}\left(\boldsymbol{R}^{N}\right)} \leq \gamma\left\|u_{0}\right\|_{L^{1}\left(\boldsymbol{R}^{N}\right)}^{\frac{2}{H}} t^{-\frac{N+1}{H}} .
$$

Here $H=(N-1)(m-1)+2 m$.

We confine ourselves to prove the a-priori sup bound for the (weak) solution, whose definition is standard. Then the existence follows via a routine regularization procedure (see [5], [11] and also [1], [3]).

The first author is member of the Gruppo Nazionale per la Fisica Matematica (GNFM) of the Istituto Nazionale di Alta Matematica (INdAM). 
Note that the known estimate (see for example [5], [11] and comments in $[2])$

$$
\|u(t)\|_{L^{\infty}\left(\boldsymbol{R}^{N}\right)} \leq \gamma\left\|u_{0}\right\|_{L^{1}\left(\boldsymbol{R}^{N}\right)}^{\frac{2}{N(1)+2}} t^{-\frac{N}{N(m-1)+2}},
$$

certainly holds for all positive times. However, it is easy to check that for $t \rightarrow+\infty$ (3) gives a faster decay in time than (4).

Remark 1.2. In the case $N=1$ (3) follows from [10]. Estimates like (3) were obtained also in [2] for a class of doubly degenerate parabolic equations. Though the cases studied in [2] do not include the case of (1), the sup estimate obtained there formally coincides with the one found here, and is proven to be sharp in [2, Subsection 1.3].

The proof of Theorem 1.1 is based on the approach of [2] and [5], relying on suitable iterative estimates of integral norms. Classically, this approach makes use of Sobolev embedding theorems. In the case of equation (1) the presence of the convective term causes a marked anisotropy in the behavior of solutions. Though this does not appear explicitly in our main estimate, we must deal with this feature and in some sense take advantage of it. This is the purpose of the entropy type inequality stated below in Lemma 2.2, where we obtain integral estimates on $(N-1)$-dimensional hyperplanes orthogonal to the convection direction.

Estimates for large times of the anisotropic behavior of solutions to diffusion-convection equations were obtained in [6], [7], [8], [9] (where diffusion is linear). The use of entropy type inequalities goes back to those papers, but we use a weaker form of entropy type inequality than the one used there.

\section{Auxiliary Results}

In the following, $u$ denotes a non-negative solution to (1).

Lemma 2.1. For any $t>0$ we have

$$
u_{t} \geq-\frac{u}{(m-1) t} \text {. }
$$

in the integral sense.

For the proof of this well known result we refer to [4] or to [11, Lemma 8.1]. Estimate (5) is used in the proof of our next Lemma.

Denote for $a>0, k>0$,

$$
G_{k}(u)=\int_{k}^{u} s_{2}^{m-1}(s-k)_{+}^{a} \mathrm{~d} s,
$$


and for $a=0, k>0$

$$
G_{k}(u)=\frac{(u-k)_{+}^{m}}{m}
$$

Lemma 2.2. For any $a>0, k>0$, for any hyperplane $\boldsymbol{R}_{x^{\prime}}^{N-1}=\left\{x_{N}=\right.$ $y\}$, where we denote by $x^{\prime}$ the variable in $\boldsymbol{R}^{N-1}$, for any $t>0$,

$$
\int_{\boldsymbol{R}_{x^{\prime}}^{N-1}} G_{k}\left(u\left(x^{\prime}, y, t\right)\right) \mathrm{d} x^{\prime} \leq(m(m-1) t)^{-1} \int_{\boldsymbol{R}^{N}} u(u-k)_{+}^{a} \mathrm{~d} x .
$$

When $a=0$ we have, denoting by $\chi_{A}$ the characteristic function of the set $A$,

$$
\int_{\boldsymbol{R}_{x^{\prime}}^{N-1}} G_{k}\left(u\left(x^{\prime}, y, t\right)\right) \mathrm{d} x^{\prime} \leq(m(m-1) t)^{-1} \int_{\boldsymbol{R}^{N}} u \chi_{\{u>k\}} \mathrm{d} x .
$$

Proof. Assume first that $a>0$. Multiply both sides of (1) by $(u-k)_{+}^{a}$ and integrate over $\boldsymbol{R}^{N-1} \times(-\infty, y)$. We get on using the bound for $u_{t}$ proved in Lemma 2.1 and integrating by parts

$$
\begin{aligned}
m \int_{-\infty}^{y} \mathrm{~d} x_{N} & \int_{\boldsymbol{R}_{x^{\prime}}^{N-1}}\left(\int_{k}^{u} s^{m-1}(s-k)_{+}^{a} \mathrm{~d} s\right)_{x_{N}} \mathrm{~d} x^{\prime} \leq \\
& m \frac{d}{d y} \int_{\boldsymbol{R}_{x^{\prime}}^{N-1}} G_{k}\left(u\left(x^{\prime}, y, t\right)\right) \mathrm{d} x^{\prime}+\frac{1}{(m-1) t} \int_{\boldsymbol{R}^{N}} u(u-k)_{+}^{a} \mathrm{~d} x,
\end{aligned}
$$

whence integrating the left hand side

$$
\begin{aligned}
& m \int_{\boldsymbol{R}_{x^{\prime}}^{N-1}} G_{k}\left(u\left(x^{\prime}, y, t\right)\right) \mathrm{d} x^{\prime} \leq \\
& \quad m \frac{d}{d y} \int_{\boldsymbol{R}_{x^{\prime}}^{N-1}} G_{k}\left(u\left(x^{\prime}, y, t\right)\right) \mathrm{d} x^{\prime}+\frac{1}{(m-1) t} \int_{\boldsymbol{R}^{N}} u(u-k)_{+}^{a} \mathrm{~d} x .
\end{aligned}
$$

Multiplying both sides of this inequality by $\exp \{-y\}$ and integrating the result between $y_{1}$ and $y_{2}$ we obtain

$$
\begin{aligned}
E_{k}\left(y_{1}\right):= & \int_{\boldsymbol{R}_{x^{\prime}}^{N-1}} G_{k}\left(u\left(x^{\prime}, y_{1}, t\right)\right) \mathrm{d} x^{\prime} \leq \exp \left\{-\left(y_{2}-y_{1}\right)\right\} E_{k}\left(y_{2}\right)+ \\
& (m(m-1) t)^{-1} \int_{\boldsymbol{R}^{N}} u(u-k)_{+}^{a} \mathrm{~d} x .
\end{aligned}
$$

Letting $y_{2} \rightarrow \infty$, we arrive at (6), for $y=y_{1}$. 
Finally the case $a=0$ follows on letting $a \rightarrow 0$ above, which is possible since the quantities involved do not critically depend on $a$.

\section{Proof of Theorem 1.1}

We denote by $\gamma>0, b>1$ constants depending only on $N, m$, which may vary from line to line.

Let us state first the following energy inequality which can be proved by means of routine calculations.

Lemma 3.1. For all $h_{1}>h_{2}>0, t>t_{1}>t_{2}>0$ and $\omega>0$ we have

$$
\begin{aligned}
\sup _{t_{1}<\tau<t} \int_{\boldsymbol{R}^{N}}\left(u-h_{1}\right)_{+}^{1+\omega} \mathrm{d} x+\int_{t_{1}}^{t} \int_{\boldsymbol{R}^{N}}\left|\nabla\left(u-h_{1}\right)_{+}^{\frac{m+\omega}{2}}\right|^{2} \mathrm{~d} x \mathrm{~d} \tau \\
\leq \frac{\gamma}{t_{1}-t_{2}} \int_{t_{2}}^{t} \int_{\boldsymbol{R}^{N}}\left(u-h_{2}\right)_{+}^{1+\omega} \mathrm{d} x \mathrm{~d} \tau .
\end{aligned}
$$

Fix $t>0$. Introduce then the decreasing sequences

$$
\vartheta_{n}=\tau_{2}+\left(\tau_{1}-\tau_{2}\right) 2^{-n}, \quad k_{n}=a_{2}+\left(a_{1}-a_{2}\right) 2^{-n}, \quad n \geq 0,
$$

for arbitrarily fixed $t>\tau_{1}>\tau_{2}>t / 4$ and $a_{1}>a_{2}>0$. Next we apply Lemma 3.1 with $t_{1}=\vartheta_{n}, t_{2}=\vartheta_{n+1}, h_{1}=k_{n}, h_{2}=k_{n+1}$. We obtain

$$
\begin{aligned}
& \sup _{\vartheta_{n}<\tau<t} \int_{\boldsymbol{R}^{N}}\left(u-k_{n}\right)_{+}^{1+\omega} \mathrm{d} x+\iint_{E_{n}} \mid \nabla\left.\left(u-k_{n}\right)_{+}^{\frac{m+\omega}{2}}\right|^{2} \mathrm{~d} x \mathrm{~d} \tau \\
& \leq \frac{\gamma b^{n}}{\tau_{1}-\tau_{2}} \iint_{E_{n+1}}\left(u-k_{n+1}\right)_{+}^{1+\omega} \mathrm{d} x \mathrm{~d} \tau,
\end{aligned}
$$

where $E_{n}=\boldsymbol{R}^{N} \times\left(\vartheta_{n}, t\right)$. Here $\omega>0$ is any fixed constant, whose value is in practice not relevant. Set $v_{n}=\left(u-k_{n}\right)_{+}^{\frac{m+\omega}{2}}$. Then the last inequality leads to

$$
\sup _{\vartheta_{n}<\tau<t} \int_{\boldsymbol{R}^{N}} v_{n}^{\beta} \mathrm{d} x+\iint_{E_{n}}\left|\nabla v_{n}\right|^{2} \mathrm{~d} x \mathrm{~d} \tau \leq \frac{\gamma b^{n}}{\tau_{1}-\tau_{2}} \iint_{E_{n+1}} v_{n+1}^{\beta} \mathrm{d} x \mathrm{~d} \tau ;
$$

we employ here the notation

$$
\beta=2 \frac{1+\omega}{m+\omega}, \quad \delta=\frac{2}{m+\omega} .
$$


On applying next Lemma 2.2 with $k=k_{n}, a=0$ we can easily get at every time level $\tau>t / 4$

$$
\int_{\boldsymbol{R}_{x^{\prime}}^{N-1}} v_{n}^{m \delta} \mathrm{d} x^{\prime} \leq \gamma b^{n} \frac{a_{1}}{a_{1}-a_{2}} t^{-1} \int_{\boldsymbol{R}^{N}} v_{n+1}^{\delta} \mathrm{d} x .
$$

Next we use the $N-1$ dimensional Nirenberg-Gagliardo type inequality

$$
\int_{\boldsymbol{R}_{x^{\prime}}^{N-1}} v_{n+1}^{\beta} \mathrm{d} x^{\prime} \leq \gamma\left(\int_{\boldsymbol{R}_{x^{\prime}}^{N-1}}\left|\nabla v_{n+1}\right|^{2} \mathrm{~d} x^{\prime}\right)^{\frac{\alpha \beta}{2}}\left(\int_{\boldsymbol{R}_{x^{\prime}}^{N-1}} v_{n+1}^{\mu^{\prime}} \mathrm{d} x^{\prime}\right)^{\frac{(1-\alpha) \beta}{\mu^{\prime}}} .
$$

where $\mu^{\prime}=(1+\mu) \delta, 0<\mu<\min (m-1, \omega)$ will be chosen and $\alpha$ by dimensional analysis is calculated as

$$
\frac{N-1}{\beta}=\alpha \frac{N-3}{2}+(1-\alpha) \frac{N-1}{\mu^{\prime}} .
$$

By the Hölder inequality with $\mu<m-1$ and by (10) we infer

$$
\begin{gathered}
\int_{\boldsymbol{R}_{x^{\prime}}^{N-1}} v_{n+1}^{\mu^{\prime}} \mathrm{d} x^{\prime} \leq\left(\int_{\boldsymbol{R}_{x^{\prime}}^{N-1}} v_{n+1}^{m \delta} \mathrm{d} x^{\prime}\right)^{\frac{\mu}{m-1}}\left(\int_{\boldsymbol{R}_{x^{\prime}}^{N-1}} v_{n+1}^{\delta} \mathrm{d} x^{\prime}\right)^{1-\frac{\mu}{m-1}} \\
\leq\left(\gamma b^{n} \frac{a_{1}}{a_{1}-a_{2}} t^{-1} \int_{\boldsymbol{R}^{N}} v_{n+2}^{\delta} \mathrm{d} x\right)^{\frac{\mu}{m-1}}\left(\int_{\boldsymbol{R}_{x^{\prime}}^{N-1}} v_{n+1}^{\delta} \mathrm{d} x^{\prime}\right)^{1-\frac{\mu}{m-1}} .
\end{gathered}
$$

Therefore by (11) we get

$$
\begin{aligned}
& \int_{\boldsymbol{R}_{x^{\prime}}^{N-1}} v_{n+1}^{\beta} \mathrm{d} x^{\prime} \leq \gamma\left(\int_{\boldsymbol{R}_{x^{\prime}}^{N-1}}\left|\nabla v_{n+1}\right|^{2} \mathrm{~d} x^{\prime}\right)^{\frac{\alpha \beta}{2}} \\
\times & \left(b^{n} \frac{a_{1}}{a_{1}-a_{2}} t^{-1} \int_{\boldsymbol{R}^{N}} v_{n+2}^{\delta} \mathrm{d} x\right)^{\frac{\mu}{m-1} \frac{(1-\alpha) \beta}{\mu^{\prime}}}\left(\int_{\boldsymbol{R}_{x^{\prime}}^{N-1}} v_{n+1}^{\delta} \mathrm{d} x^{\prime}\right)^{\left(1-\frac{\mu}{m-1}\right) \frac{(1-\alpha) \beta}{\mu^{\prime}}}
\end{aligned}
$$


Integrate (13) in $x_{N}$ between $-\infty$ and $\infty$ and apply the Hölder inequality to get

$$
\begin{aligned}
& \int_{\boldsymbol{R}^{N}} v_{n+1}^{\beta} \mathrm{d} x \leq \gamma b^{n}\left(\int_{\boldsymbol{R}^{N}}\left|\nabla v_{n+1}\right|^{2} \mathrm{~d} x\right)^{\frac{\alpha \beta}{2}} \\
& \times\left(\frac{a_{1}}{a_{1}-a_{2}} t^{-1} \int_{\boldsymbol{R}^{N}} v_{n+2}^{\delta} \mathrm{d} x\right)^{A\left(1-\frac{\alpha \beta}{2}\right)}\left(\int_{-\infty}^{\infty}\left(\int_{\boldsymbol{R}_{x^{\prime}}^{N-1}} v_{n+1}^{\delta} \mathrm{d} x^{\prime}\right)^{B} \mathrm{~d} x_{N}\right)^{1-\frac{\alpha \beta}{2}} .
\end{aligned}
$$

We have used the fact $\alpha \beta<2$ which can be computed to actually hold true according to the value of $\mu$ selected below. Here we denote

$$
A=\frac{\mu}{m-1} \frac{(1-\alpha) \beta}{\mu^{\prime}}\left(1-\frac{\alpha \beta}{2}\right)^{-1}, \quad B=\left(1-\frac{\mu}{m-1}\right) \frac{A(m-1)}{\mu}
$$

Choose now the free parameter $\mu$ by imposing the relation $B=1$. Then from (15) and (12) one gets for $K=(N-1)(m-1)+2$

$$
\mu=\frac{2 \omega(m-1)}{K+2(\omega+m-1)}<\min (m-1, \omega), \quad A=\frac{2 \omega}{K+2(m-1)}
$$

Integrating now $(14)$ in time over $\left(\vartheta_{n+1}, t\right)$ and applying the Young inequality we obtain

$$
\begin{aligned}
& \frac{b^{n}}{\tau_{1}-\tau_{2}} \iint_{E_{n+1}} v_{n+1}^{\beta} \mathrm{d} x \mathrm{~d} \tau \leq \epsilon \iint_{E_{n+1}}\left|\nabla v_{n+1}\right|^{2} \mathrm{~d} x \mathrm{~d} \tau \\
& +\gamma(\epsilon) b^{n}\left(\tau_{1}-\tau_{2}\right)^{-\frac{2}{2-\alpha \beta}}\left(\frac{a_{1} t^{-1}}{a_{1}-a_{2}}\right)^{A} \\
& \quad \times\left(t-\vartheta_{n+1}\right)\left(\sup _{\vartheta_{n+1}<\tau<t} \int_{\boldsymbol{R}^{N}} v_{n+2}^{\delta} \mathrm{d} x\right)^{1+A} .
\end{aligned}
$$


Finally, from (9) and (16) we infer

$$
\begin{aligned}
L_{n}:=\sup _{\vartheta_{n}<\tau<t} \int_{\boldsymbol{R}^{N}} v_{n}^{\beta} \mathrm{d} x+\iint_{E_{n}}\left|\nabla v_{n}\right|^{2} \mathrm{~d} x \mathrm{~d} \tau \leq \epsilon \iint_{E_{n+1}}\left|\nabla v_{n+1}\right|^{2} \mathrm{~d} x \mathrm{~d} \tau \\
\quad+\gamma_{1} b^{n}\left(\tau_{1}-\tau_{2}\right)^{-\frac{2}{2-\alpha \beta}} t^{-A}\left(\frac{a_{1}}{a_{1}-a_{2}}\right)^{A} \\
\quad \times\left(t-\vartheta_{n+1}\right)\left(\sup _{\vartheta_{n+1}<\tau<t} \int_{\boldsymbol{R}^{N}} v_{n+2}^{\delta} \mathrm{d} x\right)^{1+A} .
\end{aligned}
$$

Here we denote by $\gamma_{1}$ a constant depending on suitable powers of $\epsilon>0$.

Iterating this inequality with respect to $n$, we obtain

$$
\begin{aligned}
L_{0} \leq \epsilon^{n} L_{n}+\left(\sum_{i=0}^{n-1}(\epsilon b)^{i}\right) \gamma_{1}\left(\tau_{1}-\tau_{2}\right)^{-\frac{2}{2-\alpha \beta}} t^{-A}\left(\frac{a_{1}}{a_{1}-a_{2}}\right)^{A} & \\
& \times\left(t-\tau_{2}\right)\left(\sup _{\tau_{2}<\tau<t} \int_{\boldsymbol{R}^{N}}\left(u-a_{2}\right)_{+} \mathrm{d} x\right)^{1+A} .
\end{aligned}
$$

Therefore on selecting $\epsilon<b^{-1}$ we have as $n \rightarrow \infty$

$$
\begin{aligned}
\sup _{\tau_{1}<\tau<t} \int_{\boldsymbol{R}^{N}}\left(u-a_{1}\right)_{+}^{1+\omega} \mathrm{d} & \leq \gamma_{1}\left(\tau_{1}-\tau_{2}\right)^{-\frac{2}{2-\alpha \beta}} t^{-A}\left(\frac{a_{1}}{a_{1}-a_{2}}\right)^{A} \\
& \times\left(t-\tau_{2}\right)\left(\sup _{\tau_{2}<\tau<t} \int_{\boldsymbol{R}^{N}}\left(u-a_{2}\right)_{+} \mathrm{d} x\right)^{1+A} .
\end{aligned}
$$

For $k>0$ to be chosen define for $n \geq 0$

$$
h_{n}=k-2^{-n-1} k, \quad t_{n}=2^{-1} t-2^{-n-2} t, \quad \widetilde{h}_{n}=2^{-1}\left(h_{n}+h_{n+1}\right),
$$

and note that

$$
\left(u-h_{n+1}\right)_{+} \leq 2^{\omega(n+3)} k^{-\omega}\left(u-\widetilde{h}_{n}\right)_{+}^{1+\omega} .
$$

Therefore, on applying (17) with

$$
a_{1}=\widetilde{h}_{n}, \quad a_{2}=h_{n}, \quad \tau_{1}=t_{n+1}, \quad \tau_{2}=t_{n},
$$

so that

we obtain

$$
\frac{a_{1}}{a_{1}-a_{2}} \leq 2^{n+3}
$$

$$
Y_{n+1}:=\sup _{t_{n+1}<\tau<t} \int_{\boldsymbol{R}^{N}}\left(u-h_{n+1}\right)_{+} \mathrm{d} x \leq \gamma_{1} b^{n} k^{-\omega} t^{-\frac{\alpha \beta}{2-\alpha \beta}-A} Y_{n}^{1+A} .
$$


Finally, by means of a standard iterative lemma (see e.g., [5] p.12) we conclude that $Y_{n} \rightarrow 0$ as $n \rightarrow \infty$ provided

$$
k^{-\omega} t^{-\frac{\alpha \beta}{2-\alpha \beta}-A} Y_{0}^{A} \leq \sigma
$$

for a small enough $\sigma=\sigma(m, N)$. After a lenghty but elementary calculation (see the Appendix below) the last inequality leads to

$$
k^{-H} t^{-N-1} Y_{0}^{2} \leq \sigma^{\frac{K+2(m-1)}{\omega}} .
$$

Finally, noting that

and choosing

$$
Y_{0} \leq\left\|u_{0}\right\|_{L^{1}\left(\boldsymbol{R}^{N}\right)}
$$

$$
k=\sigma^{-\frac{K+2(m-1)}{\omega}} t^{-\frac{N+1}{H}}\left\|u_{0}\right\|_{L^{1}\left(\boldsymbol{R}^{N}\right)}^{\frac{2}{H}},
$$

we conclude the proof of Theorem 1.1.

Acknowledgments: The authors are indebted with the referees for many enhancements to the presentation.

\section{Compliance with Ethical Standards:}

CONFLICT OF INTEREST: The authors declare that they have no conflict of interest.

\section{Appendix: Calculation of the exponent in the main ESTIMATE}

We show here how (18) yields (19); this follows from (24), (25) which we prove below.

Recall our definitions

$$
\delta=\frac{2}{m+\omega}, \quad \beta=\frac{2(1+\omega)}{m+\omega}, \quad \mu^{\prime}=(1+\mu) \delta=\frac{2(1+\mu)}{m+\omega} .
$$

From the definition of $\alpha$ in (12) we obtain

$$
\alpha=\frac{\frac{N-1}{\mu^{\prime}}-\frac{N-1}{\beta}}{\frac{N-1}{\mu^{\prime}}-\frac{N-3}{2}}=\frac{2}{\beta} \frac{(N-1)\left(\beta-\mu^{\prime}\right)}{(N-1)\left(2-\mu^{\prime}\right)+2 \mu^{\prime}} .
$$

Hence

$$
\begin{aligned}
\frac{\alpha \beta}{2}=\frac{(N-1)(\omega-\mu)}{(N-1)(m-1)+(N-1)(\omega-\mu)+2(1+\mu)} & \\
= & \frac{(N-1)(\omega-\mu)}{K+(N-1)(\omega-\mu)+2 \mu} .
\end{aligned}
$$

Next we compute from (20)

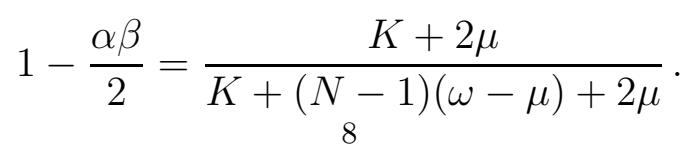


We have from the definition of $\alpha$

$$
\begin{aligned}
1-\alpha=\frac{\frac{N-1}{\beta}-\frac{N-3}{2}}{\frac{N-1}{\mu^{\prime}}-\frac{N-3}{2}}=\frac{\mu^{\prime}}{\beta} \frac{(N-1)(2-\beta)+2 \beta}{(N-1)\left(2-\mu^{\prime}\right)+2 \mu^{\prime}} \\
=\frac{\mu^{\prime}}{\beta} \frac{K+2 \omega}{K+(N-1)(\omega-\mu)+2 \mu},
\end{aligned}
$$

that is

$$
(1-\alpha) \frac{\beta}{\mu^{\prime}}=\frac{K+2 \omega}{K+(N-1)(\omega-\mu)+2 \mu} .
$$

Therefore from the definition of $A$ in (15), from (21) and (22) we get

$$
A \frac{m-1}{\mu}=(1-\alpha) \frac{\beta}{\mu^{\prime}}\left(1-\frac{\alpha \beta}{2}\right)^{-1}=\frac{K+2 \omega}{K+2 \mu} .
$$

From this equality we infer imposing that $B=1$

$$
1=B:=\left(1-\frac{\mu}{m-1}\right) A \frac{m-1}{\mu}=\frac{m-1-\mu}{m-1} \frac{K+2 \omega}{K+2 \mu} .
$$

Thus with the choice $B=1$ we solve the last equality for $\mu$ as

$$
\mu=\frac{2 \omega(m-1)}{K+2(\omega+m-1)}=\frac{2 \omega(m-1)}{H+2 \omega},
$$

and therefore (using $B=1$ again and (23))

$$
A=\frac{\frac{\mu}{m-1}}{1-\frac{\mu}{m-1}}=\frac{2 \omega}{K+2(m-1)}=\frac{2 \omega}{H} .
$$

Next we have from (20), (21) and (23)

$$
\begin{aligned}
\frac{\alpha \beta}{2-\alpha \beta}= & \frac{\alpha \beta}{2(1-\alpha \beta / 2)}=\frac{(N-1)(\omega-\mu)}{K+2 \mu}= \\
& \frac{(N-1)\left(\omega-\frac{2 \omega(m-1)}{H+2 \omega}\right)}{K+2 \frac{2 \omega(m-1)}{H+2 \omega}}=\frac{(N-1)(K+2 \omega) \omega}{H(K+2 \omega)}=\frac{(N-1) \omega}{H} .
\end{aligned}
$$

Finally from the last equality and (24) we conclude that

$$
\frac{\alpha \beta}{2-\alpha \beta}+A=\frac{(N+1) \omega}{H},
$$

so that by substituting (24), (25) in inequality (18) we get the desired result. 


\section{REFERENCES}

[1] D. Andreucci, R. Cirmi, S. Leonardi and A.F. Tedeev. Large Time Behavior of Solutions to the Neumann Problem for a Quasilinear Second Order Degenerate Parabolic Equation in Domains with Noncompact Boundary. Journal of Differential Equations, 174:253-288, 2001.

[2] D. Andreucci and A.F. Tedeev. Large time behaviour for degenerate parabolic equations with convection. Asymptotic Analysis, 60 (3-4):227-247, 2008.

[3] D. Andreucci and A.F. Tedeev. The Cauchy-Dirichlet Problem for The Porous Media Equation in Cone-Like Domains. SIAM J. on Mathematical Analysis 46:1427-1455, 2014.

[4] Ph. Bènilan and M.G. Crandall. Regularizing effects of homogeneous evolution equations. Contributions to analysis and geometry (Baltimore, Md., 1980) 2339, Johns Hopkins Univ. Press, Baltimore, Md., 1981.

[5] E. DiBenedetto. Degenerate Parabolic Equations. Springer Verlag, New York, 1993.

[6] G. Duro and E. Zuazua. Large time behaviour for convection-diffusion equations in $R^{N}$ with asymptotically constant diffusion. Comm. Partial Differential Equations, 25(1-2):73-99, 2000.

[7] M. Escobedo, J.L. Vazquez, and E. Zuazua. Asymptotic behaviour and sourcetype solutions for a diffusion-convection equation. Arch. Rational Mech. Anal., 124(1):43-65, 1993.

[8] M. Escobedo, J.L. Vazquez, and E. Zuazua. A diffusion-convection equation in several space dimensions. Indiana Univ. Math. J., 42(4):1413-1440, 1993.

[9] M. Escobedo and E. Zuazua. Long-time behaviour for convection-diffusion equation in higher dimensions. SIAM J. Math. Anal., 28(3):570-594, 1997.

[10] P. Laurencot and F. Simondon. Long-time behaviour for porous medium equations with convection. Proc. Roy. Soc. Edinburgh Sect. A, 128(2):315-336, 1998.

[11] J.L. Vazquez. The porous medium equation, Mathematical theory. Oxford Mathematical Monographs., Oxford, 2007.

Department of Basic and Applied Sciences for Engineering, Sapienza

UNIVERSITY OF ROME, ITALY

Email address: daniele.andreucci@sbai.uniroma1.it

South Mathematical Institute of VSC RaS, Vladikavkaz, Russian FEDERATION

Email address: a_tedeev@yahoo.com 